\title{
3 Definition von Schul-Amok und School Shooting
}

Die Begriffe „Schul-Amok“ und „School Shooting“ lassen sich der Bezeichnung „Massaker“ unterordnen. In Medien ist häufig auch von „Schulmassakern“ die Rede. Unter einem Massaker versteht man einen „Massenmord“, der unter besonders grausamen, man sagt auch „unmenschlichen“ Umständen durchgeführt wird. Man spricht dann auch von einem „Blutbad“. Die Menschheitsgeschichte bietet eine unübersehbare Fülle solcher entsetzlicher Ereignisse.

Im engeren Sinne fasst man Schul-Amokläufe und School Shootings unter der Bezeichnung „schwere zielgerichtete Gewalttaten an Schulen“ zusammen. Die Begriffe Schul-Amoklauf und School Shooting werden aber nicht allen Aspekten gerecht. Zum einen steht „Amok“ ursprünglich für einen plötzlichen, ungeplanten Gewaltausbruch im Gegensatz zu den hier beschriebenen, oft über einen langen Zeitraum geplanten Taten. Zum anderen werden dabei nicht zwingend Schusswaffen eingesetzt.

Im DSM-IV wird Amok unter dem Aspekt „Störungen der Impulskontrolle“ im Sinne einer „intermittierenden explosiblen Störung“ erwähnt. Dort tritt Amok aber eher als einzelne Episode und nicht als Muster aggressiven Verhaltens auf und geht oft mit ausgeprägten dissoziativen Merkmalen einher.

Im DSM-IV-TR wird Amok im „Glossar kulturabhängiger Syndrome“ beschrieben als eine

„dissoziative Episode, die durch eine Periode des Grübelns charakterisiert ist, auf die ein Ausbruch gewalttätigen, aggressiven oder Menschen gefährden- 
den Verhaltens folgt, das sich auf Personen und Objekte richtet. Eine solche Episode geht oft mit Verfolgungsideen, Automatismen, Amnesie und Erschöpfung sowie einer anschließenden Rückkehr zum prämorbiden Status einher. In einigen Fällen tritt Amok während einer kurzen psychotischen Episode auf oder kann den Beginn oder die Verschlechterung eines chronisch verlaufenden psychotischen Prozesses kennzeichnen."

(Sass et al., 2003).

Im Gegensatz zum DSM-IV empfiehlt das ICD-10 die Einordnung von Amok in das bestehende System unter Persönlichkeits- und Verhaltensstörungen (F 68.8).

Amok wird im Anhang II zum ICD-10 „Forschung und Praxis“ für Indonesien und Malaysia aufgeführt und wie folgt beschrieben:

„Eine willkürliche, anscheinend nicht provozierte Episode mörderischen oder erheblich destruktiven Verhaltens, gefolgt von Amnesie oder Erschöpfung. Viele Episoden gipfeln im Suizid.“

Wahl und Hees (2009) stellen fest, dass Schulmassaker mit den oben beschriebenen Amokläufen darin übereinstimmen, dass auch sie häufig von einzelnen männlichen Jugendlichen verübt werden. Die Ursache für ihre Gewalttaten sehen sie in vorausgegangenen verletzenden Erfahrungen von Schikanen, Status- oder Beziehungsverlusten, Abwertung oder Ausgrenzung in der Schule, wofür sie Rache nehmen wollen. Nach Spitczok von Brisinski (2009) handelt es sich um zuvor angepasste, zurückhaltende, eher freundliche, relativ unauffällige Außenseiter, die sich ausgegrenzt und herabgesetzt fühlten.

In vielen wissenschaftlichen Veröffentlichungen setzt sich in der Zwischenzeit für schulbezogene Gewalttaten der Begriff „School Shooting“ durch, wenngleich nicht alle Taten mit Schusswaffen auf Amoktaten zurückzuführen sind. Mit diesem Begriff werden Tötungen und Tötungsversuche von Jugendlichen bezeichnet, die in direkten Bezug zu einer schulischen Einrichtung begangen werden. Dieser Bezug kann sich in der Wahl der Opfer, insbesondere auch nach ihrer Funktion als Lehrer oder Schüler in der entsprechenden Bildungseinrichtung äußern.

Folgende Merkmale für School Shooting stellten Hoffmann, Roshdi und Robertz (2009) auf:

- School Shootings sind Tötungen und Tötungsversuche von Jugendlichen, die in einem direkten Bezug zu einer schulischen Einrichtung begangen werden.

- Die Täter sind in der Regel Schüler oder suspendierte oder ehemalige Schüler der betroffenen Schule. 
- School Shooter besuchen überwiegend mittlere oder höhere Schulen, haben Leistungsprobleme oder sind ihren Geschwistern in ihren Leistungen unterlegen.

- Sie haben Probleme, Anerkennung zu erlangen und sind meist Einzelgänger.

- Sie zeigen häufig eine depressive und narzisstische Problematik.

- Die Opfer sind Schüler, Lehrer und andere an der Schule beschäftigte Personen, wie der Hausmeister.

- In anderen Fällen sind nicht bestimmte Personen das Ziel, manchmal scheint es darum zu gehen, so viele Personen wie möglich zu töten.

- Die Taten werden lange im Vorfeld geplant. Sie folgen häufig bestimmten Vorbildern.

- Potenzielle jugendliche Täter entwickeln über lange Zeit intensive Gewaltfantasien und wollen sie schließlich in die Realität umsetzen.

- Zur Ausdifferenzierung ihrer Gewaltfantasien setzen sich diese Jugendlichen intensiv mit gewalthaltigen Medieninhalten auseinander.

- Kurz vor ihrer Tatumsetzung erlitten die Jugendlichen Schädigungen in ihren sozialen Beziehungen (Mobbing), die sie als sehr schwerwiegend wahrnahmen.

- In der Regel endet das Geschehen mit einem Suizid der Täter oder mit der Tötung durch Sicherheitskräfte.

\section{Nicht selten werden Schul-Amoktaten vorher angekündigt. Man spricht von einem Leaking-Phänomen.}

Adler (2010) kritisiert diese weitgehend kriminologisch geprägte Definition von School Shooting, da bei den neun deutschen Fällen zwischen 1999 und 2009 in acht Fällen ein Suizid oder eine Tötung durch Polizisten zu beklagen gewesen ist. Diese Sachverhalte gehören seiner Meinung nach jedoch nicht zur Definition „krimineller Handlungen“ und machen deutlich, dass es sich eher um Taten aus dem „Homizid-Suizid-Spektrum“ handelt. 